\title{
Improving the medical 'take sheet'
}

\author{
Oliver Reed
}

Belfast City Hospital

\begin{abstract}
The GMC states that "Trainees in hospital posts must have well organised handover arrangements, ensuring continuity of patient care[1]".

In the Belfast City Hospital throughout the day there can be multiple new medical admissions. These can be via the GP Unit, transfers for tertiary care, and transfers due to bed shortages in other hospitals. Over the course of 24 hours there can be up to four medical SHOs and three registrars that fill in the take sheet. Due to the variety of admission routes and number of doctors looking after the medical take information can be lost during handover between SHOs.
\end{abstract}

In the current format there is little room to write and key and relevant information on the medical take sheet about new and transferring patients. I felt that this handover sheet could be improved.

An initial questionnaire demonstrated that $47 \%$ found the old proforma easy to use and $28.2 \%$ felt that it allowed them to identify sick patients. $100 \%$ of SHOs and Registrars surveyed felt that it could be improved from its current form.

From feedback from my colleagues I created a new template and trialled it in the hospital. A repeat questionnaire demonstrated that $92.3 \%$ of responders felt the new format had improved medical handover and that $92.6 \%$ felt that it allowed safe handover most of the time/always.

The success of this new proforma resulted in it being implemented on a permanent basis for new medical admissions and transfers to the hospital.

\section{Problem}

Medical handover of patients is a key aspect of good clinical care. This is especially important since the introduction of the European Working Time Directive (EWTD) [2] as a doctor may have no day to day contact with the patients for whom he will care and be responsible for during an out of hours shift.

In the Belfast City Hospital, General Medicine covers seven sub -specialities which are managed on a daily basis by 24 medical Senior House Officer's (SHO's) and 16 registrars. Throughout the day there can be multiple new admissions through several routes including the GP Emergency Receiving Unit (ERU), referrals for tertiary care, and inpatient transfers due to bed shortages in surrounding hospitals. Over the course of 24 hours there can be up to four medical SHO's and three registrars that fill in the admission take sheet. Due to the variety of admission routes and a number of different doctors looking after the medical take, key information relating to each individual patient is not fully communicated during handover between SHO's.

The current format of the medical take sheet leaves insufficient space to write the important and relevant information regarding the newly admitted patients. As part of the medical take, both in and out of hours, my colleagues and I have at times felt frustrated when uncertainty arises over which patients still need to be seen, who is awaiting transfer, who is acutely unwell, and the outstanding jobs on different wards.
I therefore felt that the current format of the take sheet was contributing to poor handover and could inadvertently affect the safety of patient care, especially in the out of hours setting.

\section{Background}

As part of the General Medical Council (GMC) guidance on hospital and speciality training "Trainees in hospital posts must have well organised handover arrangements, ensuring continuity of patient care at the start and end of periods of day or night duties every day of the week." [1]. This was further endorsed by the National Patient Safety Agency (NPSA) when they advised that "clinically unstable patients are known to the senior and covering clinicians, junior members of the team are adequately briefed on concerns from previous shifts, tasks not yet completed are clearly understood by the incoming team"[3].

Both of these documents help to illustrate the importance of an appropriate medical handover. Further studies with regards to the recording of data for medical handover have shown that "over a typical weekend....only $2.5 \%$ of information from the first handover is retained at the final handover if there is no written record. If notes are taken, $85.5 \%$ of information is retained, but this rises to $99 \%$ when a standardised proforma is used"[4]. It is therefore essential that a well established and easy to use proforma is in place in any clinical environment to ensure good handover and continuity of care. This is a cornerstone of good handover and has been the focus of many Quality Improvement Projects[5][6]. 


\section{Baseline Measurement}

After discussing this problem with my colleagues the current take sheets were assessed over a period of two weeks to see how well they were completed. During this time there were 208 patient admissions/transfers recorded. During this review it was noted that key pieces of information that should be part of an admission/transfer were missing, e.g. $36.8 \%$ of patients had no documented age, $38.9 \%$ had no documented speciality triage, and in only $72.5 \%$ of admissions a place of transfer was listed. In $4.5 \%$ of patients there was no documented history at all and I observed that in $21.6 \%$ of admissions there was insufficient space to write jobs for follow up.

After this period of assessment I designed and implemented a questionnaire for the Medical SHOs to complete. This was left in the GP admission ward for two weeks and had 17 responders from the 24 Medical SHOs (70.8\%). The format of this questionnaire included yes/no questions, Likert scales in order to assess different characteristics of the take sheet and space to write suggestions for improvement.

The results of the initial questionnaire confirmed certain failings of the medical take sheet that had previously been voiced amongst the SHOs. $47 \%$ found it easy to use, only $28.2 \%$ felt that it allowed them to identify sick patients, and $38.8 \%$ felt that it allowed them to identify outstanding jobs. $58.8 \%$ felt that it delivered safe handover "some of the time" and of all the SHOs and Registrars surveyed, $100 \%$ felt that the take sheet could be improved.

From the suggestion box the most common issues raised were that there was not enough space in the current format to identify sick patents, space for outstanding jobs and space for an adequate history of presenting complaint.

See supplementary file: ds2521.docx - "Daily Take Sheet Questionaire - Pre Change (with results) \& Old Proforma"

\section{Design}

The planned intervention was to create a new medical admission/transfer proforma that encompassed most of the mandatory elements required by local trust guidelines but was easier to use, interpret, and allow a safer handover of patients. The current sheet had initially been designed using local trust guidelines and in conjunction with the Royal College of Physicians Hospital Admission Audit Tool[7].

After going through the data from my initial audit of the old take sheets and receiving feedback from the first questionnaire from my colleagues relating to the current format, I then began re-designing a new proforma. I liaised with the Consultants and Medical Staff based in the ERU to ensure they were happy with the functionality of the new format and that the new design met Trust guidelines for ongoing local audit purposes.

Once completed, the first version of the new proforma was uploaded to the local computer drive in the ERU where handover takes place at 09:00 and 17:00. This replaced the old format on the designated drive so that the medical SHOs and Registrars would have no difficulty in locating the new template to print it off thus making it accessible to everyone.

Following this change an email was sent through the Local Trust servers to inform the Medical Staff of the new proforma and that ongoing feedback and review would take place to further modify and improve the handover sheet over the upcoming weeks.

\section{Strategy}

First Plan, Do, Study, Act (PDSA) Cycle

In the first PDSA Cycle I designed a questionnaire which incorporated key areas that I felt needed to be initially addressed to establish the problem areas of the current proforma. Responders were asked to assess the proforma with regards to: being easy to use and interpret, organised, identify sick patients, identify outstanding jobs, space to write, and identifying patients not clerked in. The questionnaire also asked the responders if they felt that the proforma allowed for safe handover of patients and how it could be improved.

This questionnaire was left for a two week period in the ERU in order to collect responses. After this period responses were collected from $17 \mathrm{SHOs}(70.8 \%)$ which provided a base line of what the staff assessment of the underlying proforma as well as suggestions of what they would like to see included in the new format. After liaising with the clinical leads in the ERU to agree with my new template, I implemented the first draft of the new proforma one week after collating the results of the first questionnaire and making the changes to the old format that the junior doctors had requested.

This new sheet then replaced the previous template that had existed on the local computer drive in the ERU for ease of access. Once changed the SHOs and Registrars that were based in the Unit were contacted informing them of the new template and where to find it.

\section{Second PDSA Cycle}

After a period of two weeks the second PDSA cycle was commenced, which aimed to build on the change from the previous cycle by further improving the new take sheet. I designed a new questionnaire to compare the new format directly with the old format. This repeat questionnaire was left in the ERU and a repeat email was again sent out to the Medical SHOs and Registrars asking them to fill it in for continued feedback.

After two weeks the results were collected; 13 out of the 24 (54.2\%) SHOs responded to the questionnaire assessing the new sheet and its ability to address problems, giving feedback and further suggestions for improvement. After liaising again with the clinical leads in the ERU the proforma was further modified making further changes that the Junior Doctors had suggested in order to improve 
its functionality.

The new proforma was once again placed on the local computer drive in the ERU for ease of access. Once I had performed this change I again emailed the SHOs and Registrars that were based in the Unit to inform them that the template had changed for a final time and where they could find the new proforma.

See supplementary file: ds2599.docx - "Daily Take Sheet Questionaire - Post Change (with results) \& New Proforma"

\section{Post-measurement}

The results of the questionnaire showed that $92.3 \%$ of responders felt that the new format of the proforma had improved medical handover and that the new sheet allows safe handover most of the time / always ( $46.2 \%$ respectively).

In the key areas of review (ease of use, organised, space to write, identifying sick patients, jobs, and not clerked in) the responder satisfaction had markedly increased to over $80 \%$, whereas in the initial questionnaire all of these areas had scored less than $50 \%$ see attached results.

The majority of responders felt that the medical take sheet could not be further improved (84.6\%), however many of the SHOs in the feedback comments and in discussions with myself felt that handover would be further improved if the new sheet was filled in more appropriately and thoroughly by the staff during the day and out of hours.

See supplementary file: ds3113.docx - "Questionnaire and PDSA Resuts"

\section{Lessons and Limitations}

Overall I feel that this project was a success, however I did discover key limitations from feedback obtained from my colleagues. The overall feeling was that the new format was more fit for purpose as a handover sheet, however the issue was with how well that it was being filled in.

Many of my colleagues felt that during the day, or in out of hours care, key clinical information was not being adequately recorded on the proforma. This was felt not to be a problem of the proforma but due to the staff member not putting enough clinical information on the sheet. This issue has been addressed several times and had further been reinforced to my colleagues.

Further issues with handover in this project were that patients are, on numerous occasions, transferred over to the Hospital with no formal handover from Doctor to Doctor. This has been highlighted as an unsafe practice and the proforma has since been further modified for audit purposes to give an indication of how often this is occurring.

Issues with the quality improvement project itself include: due to the assessment of each PDSA cycle change relying on \% satisfaction, there is potential for bias in response rates from the responders. Furthermore unfortunately there were a fewer number of responders in the second PDSA cycle (13 vs.17) which may have also affected results. I was also unfortunately unable to collect postintervention data to compare to pre-intervention data and complete a third PDSA cycle to further assess my final changes due to a geographic change in location of employment.

\section{Conclusion}

The introduction of the new medical take proforma has overall been a success. This was shown in the five key areas that I identified with overall satisfaction achieving at least $80 \%$ in each of them. Furthermore, $92.3 \%$ of Doctors felt that the new proforma had improved handover.

The new handover sheet also allows additional and relevant clinical information to be handed over in a safe, efficient, and effective manner in order to provide good continuity of care.

Handover can always be further improved. I have reinforced this to my colleagues by encouraging them to take the time to fill in the handover sheet more thoroughly to ensure a safe handover of patients at all times. I hope that the process of handover and will be further assessed in the future in order to find new ways to further improve it and with that patient safety.

\section{References}

1. 1. General Medical Council. The Trainee Doctor. London. General Medical Council. 2011

2. Directive $2003 / 88 / E C$ of The European Parliament and Of The Council of 4 November 2003 concerning certain aspects of the organisation of working time. Official Journal of the European Union. 2003 Nov 18; L 299/9

3. National Patient Safety Agency. Safe handover: safe patients. Guidance on clinical handover for clinicians and managers. London. National Patient Safety Agency. 2004

4. Bhabra G, Mackeith S, Monteiro P, Pothier D. An experimental comparison of handover methods. Ann R Coll Surg Engl 2007;89:298-300

5. Perry J, Manghnani R, Sommerlad A et al. Improving handovers across a North London Mental Health Trust. BMJ Qual Improv Report [online]. 2013 Oct 28 [cited 2014 Apr 22];Available from:http://qir.bmi.com/content/2/2/u707.w551.full.pdf+html

6. Curtis O, Fisher R. Improving medical SHO weekend handover at a tertiary referral centre. BMJ Qual Improv Report [online]. 2013 Aug 27 [cited 2014 Apr 22];Available from:http://qir.bmj.com/content/2/1/u697.w971.full.pdf+html

7. Royal College of Physicians: Hospital Admission Audit Tool [Online]. 2011 May 16; Available from: URL: http://www.rcplondon.ac.uk/sites/default/files/documents/hos pital-admission-clerking-audit-tool $0 . x \mid s$ 


\section{Declaration of interests}

Nothing to declare

\section{Acknowledgements}

Many thanks to the ERU team on Ward $5 \mathrm{~N}$ Belfast City Hospital for all your support. 